\title{
Finanzierung professioneller Dolmetschleistungen immer noch ungeklärt
}

\section{Lena Emch-Fassnacht}

Lic. phil., Verantwortliche Kommunikation und Projekte, INTERPRET

\author{
Zurzeit werden Dolmetschleistungen im ambulanten Bereich nicht von der öffent- \\ lichen Hand finanziert. Insbesondere für Haus- und Kinderärztinnen und -ärzte \\ ist dies problematisch. Sie müssen Verständigungsschwierigkeiten und damit \\ schlimmstenfalls eine Fehlversorgung von Patientinnen und Patienten in Kauf \\ nehmen.
}

\begin{abstract}
Die Vorteile des Einsatzes von professionellen Dolmetschenden anstelle kaum qualifizierter Übersetzungshilfen sind allgemein anerkannt: Professionelle Dolmetschende unterliegen der Schweigepflicht, sie handeln überparteilich, dolmetschen beidseitig, vollständig und sinngenau. Dies ermöglicht eine effiziente und effektive Erhebung und Kommunikation von Diagnosen, die Patientensicherheit und Wahrung der Patientenrechte, erhöht die Therapietreue und stellt einen diskriminierungsfreien Zugang zur medizinischen Grundversorgung sicher. In der Schweiz können Ärztinnen und Ärzte mit Hilfe des national anerkannten Qualifizierungssystems von INTERPRET auf professionelle Dolmetschende zurückgreifen, wenn die sprachliche Verständigung mit Patientinnen und Patienten erschwert ist [1].

Ärztinnen und Ärzte verlassen sich jedoch in vielen, auch schwierigen Situationen auf «Hände und Füsse» oder greifen auf private Übersetzungshilfen zurück. Sie tun dies mehrheitlich aus zwei Gründen:
\end{abstract}

\section{Eine Interessengemeinschaft für Verständigung}

INTERPRET ist ein 1999 in der Schweiz gegründeter unabhängiger Verein für interkulturelles Dolmetschen und Vermitteln. Er verfügt über ein national anerkanntes Ausbildungs- und Qualifizierungssystem und ist Träger des eidgenössischen Fachausweises für interkulturell Dolmetschende und Vermittelnde. Als nationale Interessengemeinschaft vereinigt und vertritt er alle Akteure des interkulturellen Dolmetschens und Vermittelns: die interkulturell Dolmetschenden, die regionalen Vermittlungsstellen sowie die Ausbildungsinstitutionen. Eine Qualifizierungsstelle definiert und überwacht Standards für die Ausbildung und Zertifizierung.
Erstens, weil die Finanzierung von professionellen Dolmetschleistungen im Gesundheitswesen nach wie vor nicht geklärt ist, und zweitens, weil die organisatorischen Hürden offenbar so hoch sind, dass die oft einfachere (Not-)Lösung vorgezogen wird. In beiden Fällen wird der Entscheid aber nicht aus fachlichen und qualitätsbezogenen Überlegungen gefällt, sondern aufgrund hinderlicher Rahmenbedingungen. Diese negativen Rahmenbedingungen gilt es zu ändern.

\section{Die aktuelle Situation}

Im Jahr 2019 wurden 163153 Dolmetscheinsätze (oder 53\% aller im Kontext des interkulturellen Dolmetschens erfassten Einsätze) im Bereich Gesundheit getätigt [2].

Von diesen rund 163000 Einsätzen fanden $28 \%$ im stationären Bereich statt. Hier empfiehlt die Konferenz der kantonalen Gesundheitsdirektorinnen und -direktoren (GDK), die Kosten für Übersetzungs- und Dolmetschdienste, welche zur Durchführung einer zweckmässigen Behandlung erforderlich sind, den Leistungen der obligatorischen Krankenpflegeversicherung (OKP) zuzurechnen [3]. Das Bundesamt für Gesundheit (BAG) und der Bundesrat unterstützen diese Empfehlung [4, 5]. Im Rahmen der Tarifautonomie liegt es jedoch im Ermessen der Tarifpartner, die Kosten der Dolmetschdienste einzuberechnen. Eine Konkretisierung hierzu fand bis jetzt nicht statt. Spitäler, Universitätskliniken und Psychiatrien behelfen sich mit unterschiedlichen Lösungen, zum Beispiel über Leistungsverträge mit den Kantonen, mit Global- oder 
Dolmetscheinsätze im Gesundheitswesen 2019

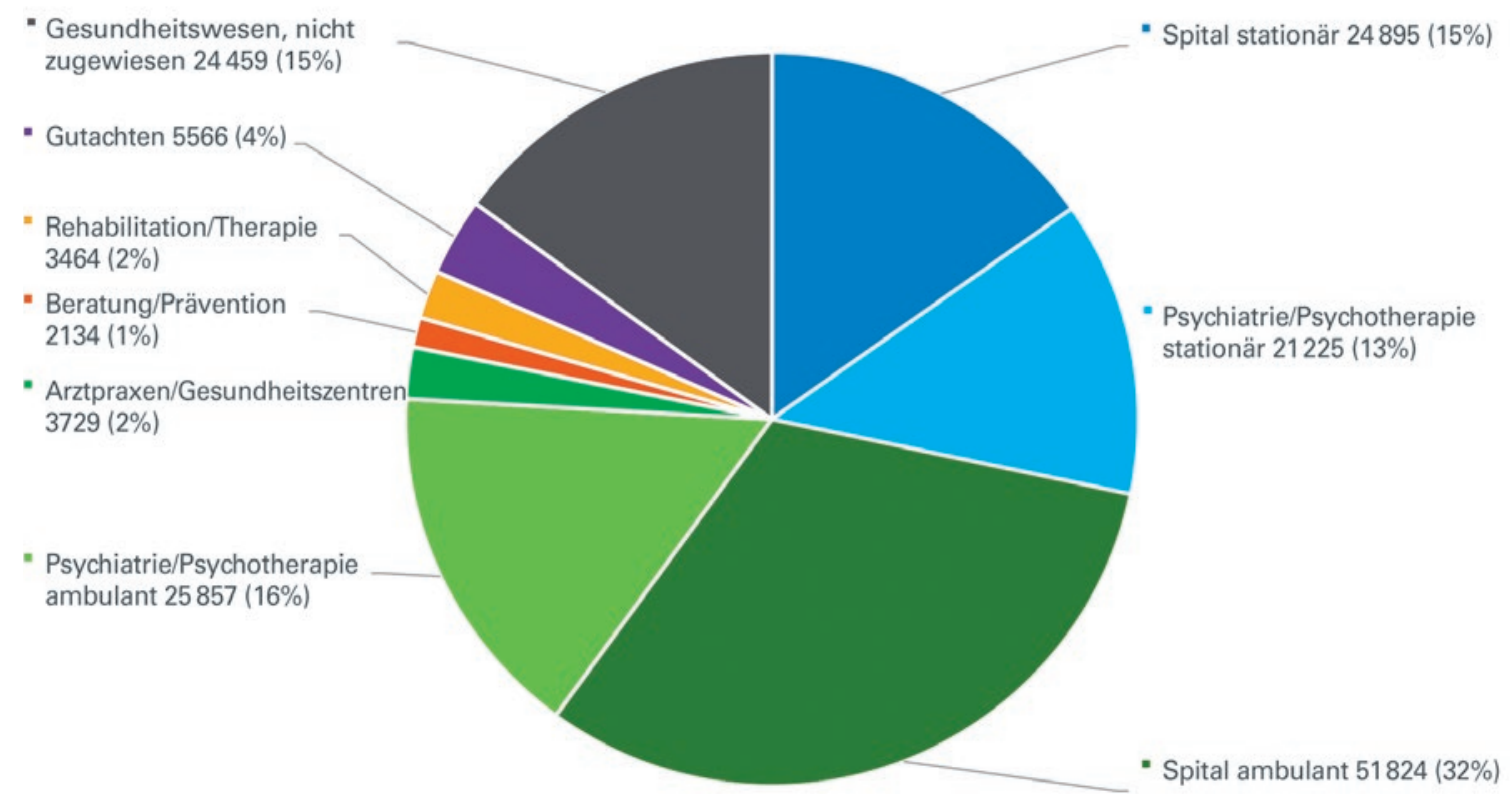

Abbildung 1: Dolmetscheinsätze im Gesundheitswesen, Stand 2019 (Quelle: INTERPRET).

Abteilungsbudgets der Institutionen oder mit zusätzlichen Fonds.

Über die Hälfte (57\%) der Dolmetscheinsätze findet jedoch im ambulanten Bereich statt. Für den grössten Teil dieser Dolmetschleistungen ist aktuell keine Finanzierung vorgesehen (Spital und Psychiatrie ambulant, Psychotherapie, private Arztpraxen). Die Kosten werden mehrheitlich von den stationären Einrichtungen (Spitäler und Psychiatrien) sowie durch private oder öffentlich-rechtliche Organisationen (z.B. Ambulatorien des Schweizerischen Roten Kreuzes, Beratungsstellen) getragen.

\section{Unbefriedigende Lage für private Praxen}

Sprachschwierigkeiten sind ein zentrales Thema in der Arzt-Patient-Interaktion [7-9]. Eine Situationsanalyse zur Lage von Hausärztinnen und -ärzten zeigt, dass für 83\% der Befragten Sprachschwierigkeiten eine besondere Herausforderung darstellen [7]. Die behelfsmässigen Lösungen, wie zum Beispiel Unterhaltung «mit Händen und Füssen» oder der Einsatz von Verwandten und Bekannten als Übersetzungshilfen, werden von den befragten Hausärztinnen und -ärzten als problematisch eingeschätzt. Denn die Schwierigkeiten bleiben damit auf beiden Seiten bestehen: «Einerseits können manche Patienten/-innen ihre Anliegen kaum ausdrücken. Andererseits ist es als Ärztin/Arzt schwierig, solche Patienten/-innen zu verstehen und Diagnosen zu stellen sowie Behandlungsschritte einzuleiten, insbesondere wenn die Patienten/-innen für längerfristige Therapien instruiert werden müssen» [7]. Hinzu kommt, dass Ärztinnen und Ärzte wegen der unübersichtlichen Lage aufgrund der unterschiedlichen Zuständigkeiten in Kantonen und Gemeinden noch viel weniger auf die Unterstützung durch profes-

Für 83\% der Hausärztinnen und Hausärzte stellen Sprachschwierigkeiten eine besondere Herausforderung dar.

sionelle Dolmetschende zurückgreifen [9]. Aus Sicht der Autorenschaft der zitierten Artikel wäre daher eine grundsätzliche Lösung der Finanzierung von Dolmetschleistungen auf nationaler Ebene wünschenswert [7, 9]. Die statistischen Auswertungen der Dolmetscheinsätze zeigen die bedenklichen Auswirkungen des derzeitigen Finanzierungsproblems: Gesamtschweizerisch fanden im Jahr 2019 in privaten Arztpraxen oder Gesundheitszentren nur 3729 Dolmetscheinsätze statt (2\%). Die Hausarztmedizin als Grundversorgung des schweizerischen Gesundheitssystems kann so einem beachtlichen Teil der Bevölkerung nicht gerecht werden.

Ein noch dramatischeres Bild zeigt sich bei der psychotherapeutischen Versorgung. So leiden zum Beispiel 40-50\% aller geflüchteten Menschen unter Traumafolgeerkrankungen, welche jedoch mehrheitlich aus sprachlichen Gründen nicht therapiert werden können [10]. Durch eine Deckung der Dolmetschkosten 
hätten traumatisierte Geflüchtete rascher Zugang zur Therapie, denn geeignete Psychotherapeutinnen und -therapeuten können auch in Privatpraxen und in der Regelversorgung Traumatherapien anbieten für geflüchtete Personen ohne ausreichende Kenntnisse der

\section{Manche Patientinnen und Patienten können ihre Anliegen kaum ausdrücken.}

Landessprachen. Dies würde die Chance auf Genesung erhöhen und das Risiko von Chronifizierungen senken, was neben hohen gesundheitlichen und gesellschaftlichen Folgekosten auch viel persönliches Leid ersparen würde.

\section{Nationale Lösungen erforderlich}

Das Problem der ungelösten Finanzierung wird von den Tarifpartnern und dem Gesetzgeber anerkannt, es herrscht aber Uneinigkeit, wer für die Lösung zuständig ist. Im Sinne einer Gleichbehandlung für alle Regionen und Leistungsbereiche fordern deshalb das Schweizerische Rote Kreuz (SRK) und INTERPRET für den ambulanten Bereich eine einheitliche nationale Lösung [11]. Der berufliche Alltag im Gesundheitswesen ist geprägt von Begegnungen. Ratsuchende zu verstehen, um gemeinsam der Situation angepasste Massnahmen zu wählen und umzusetzen, ist ein wesentlicher Teil der Arbeit und darf nicht an einer fehlenden Finanzierungslösung scheitern.

Bildnachweis

(c) INTERPRET
Literatur

1 Interpret. Ausbildung und Qualifizierung von interkulturell Dolmetschenden und Vermittelnden. www.inter-pret.ch $\rightarrow$ Ausbildung und Qualifizierung

2 Interpret. Einsatzstatistiken zum interkulturellen Dolmetschen und Vermitteln 2019; 2020. www.inter-pret.ch $\rightarrow$ Service $\rightarrow$ Statistiken

3 Konferenz der kantonalen Gesundheitsdirektorinnen und -direktoren. Empfehlungen zur Wirtschaftlichkeitsprüfung. 2019. www. gdk-cds.ch $\rightarrow$ gdk $\rightarrow$ themen $\rightarrow$ spitalfinanzierung

4 Bundesamt für Gesundheit BAG. Faktenblatt. Finanzierung des interkulturellen Dolmetschens im Gesundheitswesen durch die obligatorische Krankenpflegeversicherung (OKP). 2019.

5 Stellungnahmen des Bundesrates zur Motion 19.4279 (Sibel Arslan); 2019. www.parlament.ch/de/ratsbetrieb/suche-curiavista/geschaeft?AffairId=20194279

6 Bundesamt für Statistik BFS. Schweizerische Gesundheitsbefragung 2017; 2020. www.bfs.admin.ch/bfs/de/home/aktuell/neueveroeffentlichungen.assetdetail.14817585.html

7 Oetterli M, Laubereau B, Krongrava P, Essig S, Studer C. Unterstützung von Hausärzten/-innen bei der Behandlung von Patienten/ -innen mit Migrationshintergrund: Situationsanalyse, Handlungs bedarf und Empfehlungen zu Massnahmen. Studie zuhanden des BAG und der Dienststelle Soziales und Gesellschaft (DISG) des Kantons Luzern, Interface Politikstudien Forschung Beratung und Institut für Hausarztmedizin und Community Care. Luzern; 2016. www.bag.admin.ch $\rightarrow$ bag $\rightarrow$ empfehlungen-hausaerzte

8 Migrant Women's Health Care Needs for Chronic Illness Services in Switzerland (MIWOCA). 10 Empfehlungen zur Verbesserung der gesundheitlichen Versorgung von Frauen mit chronischen Erkrankungen unter besonderer Berücksichtigung von Patientinnen mit Migrationserfahrung. Empfehlungen. Nationales Forschungsprogramm 74 Gesundheitsversorgung, Universität Bern; 2020. www. miwoca.ch/wp-content/uploads/sites/12/2020/03/MIWOCA-Empfehlungen_DE_FINAL.pdf

9 Jäger F, Pellaud N, Laville B, Klauser P. Barriers to and solutions for addressing insufficient professional interpreter use in primary healthcare. BMC Health Services Research. 2019; 19:753.

10 Kiselev N, Morina N, Schick M, Watzke B, Schnyder U, Pfaltz M. Barriers to access to outpatient mental health care for refugees and asylum seekers in Switzerland: the therapist's view. BMC Psychiatry. 2020; 20:378.

11 Interpret, SRK. Finanzierung notwendiger Dolmetschdienste im Gesundheitswesen. 2020. www.inter-pret.ch $\rightarrow$ Positionen und Stellungnahmen
Lena Emch-Fassnacht INTERPRET

Schweizerische Interessengemeinschaft für interkulturelles Dolmetschen und Vermitteln Monbijoustrasse 61 CH-3007 Bern Tel. 0313513828 lena.emch[at]inter-pret.ch

\section{Das Wichtigste in Kürze}

- Professionelle Dolmetschende ermöglichen eine effiziente und effektive Erhebung und Kommunikation von Diagnosen, die Patientensicherheit und Wahrung der Patientenrechte, erhöhen die Therapietreue und stellen einen diskriminierungsfreien Zugang zur medizinischen Grundversorgung sicher.

- Die Finanzierung von professionellen Dolmetschleistungen im Gesundheitswesen ist nach wie vor nicht geklärt.

- Besonders im ambulanten Bereich, bei Hausarztmedizin und Psychotherapie wird aufgrund ungeklärter Finanzierungsfragen zu selten auf Dolmetschdienste zurückgegriffen.

- Das Problem der ungelösten Finanzierung wird von den Tarifpartnern und dem Gesetzgeber anerkannt, es herrscht aber Uneinigkeit, wer für die Lösung zuständig ist. Wünschenswert ist eine nationale Lösung.

\section{L'essentiel en bref}

- Les interprètes professionnels permettent une collecte et une communication efficaces et efficientes des diagnostics, la sécurité des patients et la préservation de leurs droits, augmentent I'adhésion au traitement et assurent un accès non discriminatoire aux soins de santé primaires.

- Le financement des services d'interprétation professionnelle dans le domaine de la santé n'est toujours pas réglé.

- Ce problème affecte particulièrement le secteur ambulatoire, la médecine de famille et la psychothérapie, où le recours aux services d'interprétation est trop rare.

- Le problème du financement est reconnu par les partenaires tarifaires et le législateur, mais il y a désaccord sur la question de savoir qui est chargé de trouver une solution. Une solution nationale est souhaitable. 